\title{
Leptonic decay constants for D-mesons from 3-flavour CLS ensembles
}

\author{
Sara Collins ${ }^{1}$, Kevin Eckert ${ }^{2, \star}$, Jochen Heitger ${ }^{2}$, Stefan Hofmann ${ }^{1}$, and Wolfgang Söldner ${ }^{1}$ \\ ${ }^{1}$ Universität Regensburg, Institut für Theoretische Physik, 93040 Regensburg, Germany \\ ${ }^{2}$ Westfälische Wilhelms-Universität Münster, Institut für Theoretische Physik, Wilhelm-Klemm-Straße 9, \\ 48149 Münster, Germany
}

\begin{abstract}
We report on the status of an ongoing effort by the RQCD and ALPHA Collaborations, aimed at determining leptonic decay constants of charmed mesons. Our analysis is based on large-volume ensembles generated within the CLS effort, employing $N_{\mathrm{f}}=2+1$ non-perturbatively $O(a)$ improved Wilson quarks, tree-level Symanzikimproved gauge action and open boundary conditions. The ensembles cover lattice spacings from $a \approx 0.09 \mathrm{fm}$ to $a \approx 0.05 \mathrm{fm}$, with pion masses varied from 420 to $200 \mathrm{MeV}$. To extrapolate to the physical masses, we follow both the $\left(2 m_{1}+m_{\mathrm{s}}\right)=$ const. and the $m_{\mathrm{s}}=$ const. lines in parameter space.
\end{abstract}

\section{Introduction and computational setup}

The pseudoscalar decay constants $f_{\mathrm{D}}$ and $f_{\mathrm{D}_{\mathrm{s}}}$ encode the QCD contributions in leptonic decays of Dand $\mathrm{D}_{\mathrm{s}}$-mesons, respectively. Theory input on the decay constants in conjunction with experimental data allows the CKM matrix elements $V_{\mathrm{cd}}$ and $V_{\mathrm{cs}}$ to be tightly constrained. Apart from being an important test of the Standard Model, hints of new physics may be discovered in the charm sector. For an overview of lattice QCD results, see Ref. [1]. An update on last year's report [2] and our ongoing effort on the computation of $f_{\mathrm{D}_{(\mathrm{s})}}$ is presented in the following.

We utilize ensembles generated within the Coordinated Lattice Simulations (CLS) effort, with $N_{\mathrm{f}}=2+1$ non-perturbatively $O(a)$ improved Wilson-Sheikholeslami-Wohlert (clover) fermions and tree-level improved Lüscher-Weisz gauge action, employing the openQCD [3] open-source software package. Open boundary conditions in temporal direction are used in order to avoid topological freezing, making the use of very fine lattice spacings of $a \approx 0.0854-0.039 \mathrm{fm}(\beta=3.4-3.85)$ feasible $[4,5]$. We also employ twisted-mass reweighting for light quarks [6], to prevent instabilities resulting from accidental near-zero eigenmodes of the Dirac operator, and rational approximation for the strange quark with appropriate reweighting. Expectation values of physical observables are therefore obtained by

$$
\langle O\rangle=\frac{\left\langle O W_{0} W_{1}\right\rangle_{W}}{\left\langle W_{0} W_{1}\right\rangle_{W}} .
$$

The twisted-mass $\left(W_{0}\right)$ and rational approximation reweighting $\left(W_{1}\right)$ factors are defined in Ref. [7] (Eqs. (3.2) and (3.5), respectively).

\footnotetext{
^ Speaker, e-mail: k.eckert@uni-muenster.de
} 
Apart from additional ensembles for $\beta=3.4$ and $\beta=3.55$, our analysis now also includes ensembles for the lattice spacings with $\beta=3.46, \beta=3.7$ and $\beta=3.85$. We follow two lines in the light and strange quark mass plane: (i) The average lattice quark mass $\left(\bar{m}=\left(2 m_{1}+m_{\mathrm{s}}\right) / 3\right)$ is kept fixed such that the sum of the renormalized quark masses is constant up to $O(a)$ effects (ensembles available for all $\beta$ values). (ii) The renormalized strange quark mass is kept constant, again up to $O(a)$ effects (ensembles available for $\beta=3.4$ and $\beta=3.55$ ). For details on how an almost constant renormalized strange quark mass was achieved, see Ref. [8]. We only mention that the vector Ward identity masses are defined as

$$
m_{\mathrm{q}=1(\text { ight }) \mathrm{s}}=\left(1 / \kappa_{\mathrm{q}}-1 / \kappa_{\mathrm{crit}}\right) /(2 a)
$$

where $\kappa_{\text {crit }}$ is the critical hopping parameter value at which the axial Ward identity (i.e., PCAC) quark mass in the symmetric limit, $m_{1}=m_{\mathrm{s}}$, vanishes.

The two lines are referred to as $\bar{m}=$ const. line (first proposed in [9]) and $\hat{m}_{\mathrm{s}}=$ const. line, respectively, and exemplarily visualized in Fig. 1 for the ensembles with $\beta=3.4$. Note that the figure displays the PCAC strange quark mass $\tilde{m}_{\mathrm{s}}$, for which the tuning to constant values was actually done but which corresponds to constant $\hat{m}_{\mathrm{S}}$ up to very small corrections. Having two lines in the quark mass plane available enables us to tightly constrain the chiral extrapolation, by enforcing extrapolations along both lines to intersect at the physical point. For further details concerning the computational setup see Refs. $[2,7,8,10]$.

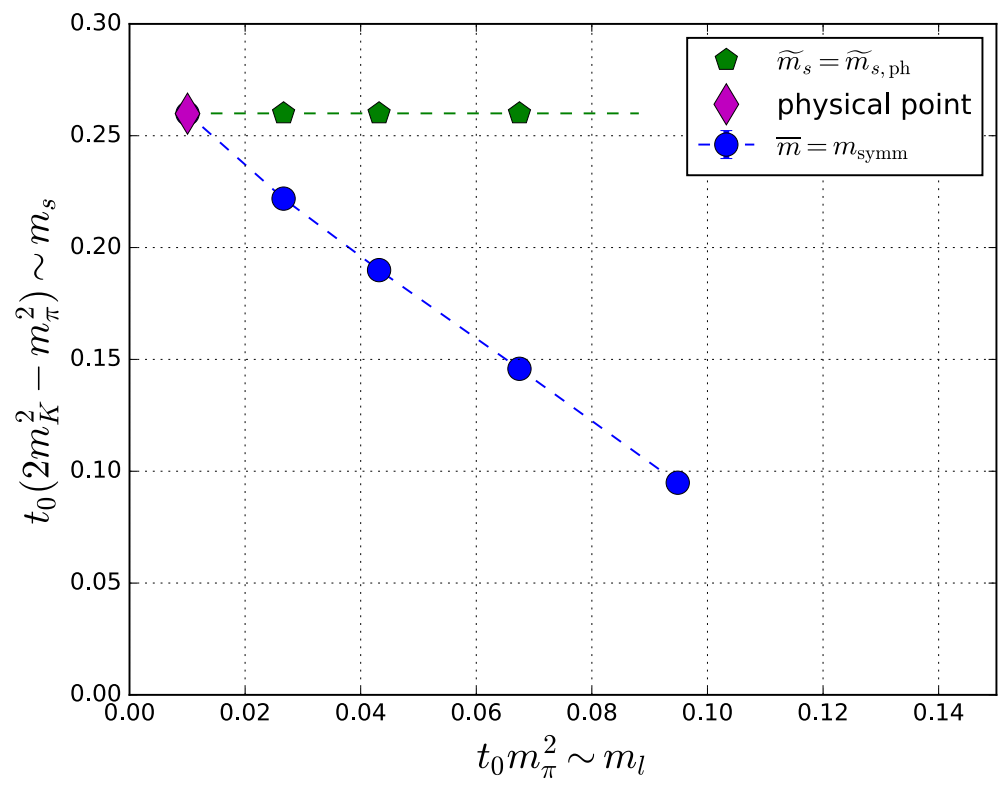

Figure 1. The light and strange quark masses realized for $\beta=3.4$ lattices as indicated by the square of the pion mass versus the kaon-pion mass difference, $2 m_{K}^{2}-m_{\pi}^{2}$, in units of the gradient flow scale parameter $t_{0}[7,8,11]$. Pion masses here vary from 422 to $223 \mathrm{MeV}$. 


\begin{tabular}{|c|c|c|c|c|c|c|c|c|}
\hline trajectory & ensemble & $\kappa_{1}$ & $\kappa_{\mathrm{s}}$ & $L m_{\pi}$ & $N_{t} \times N_{s}^{3}$ & $\frac{m_{\pi}}{\mathrm{MeV}}$ & $\frac{m_{K}}{\mathrm{MeV}}$ & $N_{\mathrm{MD}}$ \\
\hline \multicolumn{9}{|c|}{$\beta=3.4[a=0.0854(15) \mathrm{fm}], \quad \sqrt{8 t_{0}} / a=4.852(7)$} \\
\hline \multirow{3}{*}{$\bar{m}=m_{\mathrm{sym}}$} & H101 & 0.13675962 & 0.13675962 & 5.8 & $96 \times 32^{3}$ & 422 & 422 & 8000 \\
\hline & $\mathrm{H} 102$ & 0.136865 & 0.136549339 & 4.9 & $96 \times 32^{3}$ & 356 & 442 & 7988 \\
\hline & $\mathrm{H} 105$ & 0.13697 & 0.13634079 & 3.9 & $96 \times 32^{3}$ & 282 & 467 & 11332 \\
\hline \multirow{2}{*}{$\hat{m}_{\mathrm{s}}=\hat{m}_{\mathrm{s}}^{\text {(phys) }}$} & H107 & 0.136945665908 & 0.136203165143 & 5.1 & $96 \times 32^{3}$ & 368 & 549 & 6256 \\
\hline & H106 & 0.137015570024 & 0.136148704478 & 3.8 & $96 \times 32^{3}$ & 272 & 519 & 6212 \\
\hline \multicolumn{9}{|c|}{$\beta=3.46[a \approx 0.077 \mathrm{fm}], \quad \sqrt{8 t_{0}} / a \approx 5.43$} \\
\hline \multirow[b]{2}{*}{$\bar{m}=m_{\mathrm{sym}}$} & S400 & 0.136984 & 0.136702387 & 4.3 & $128 \times 32^{3}$ & 347 & 437 & 6968 \\
\hline & N401 & 0.1370616 & 0.1365480771 & 5.3 & $128 \times 48^{3}$ & 282 & 456 & 4400 \\
\hline \multicolumn{9}{|c|}{$\beta=3.55[a=0.0644(11) \mathrm{fm}], \quad \sqrt{8 t_{0}} / a=6.433(6)$} \\
\hline \multirow{5}{*}{$\bar{m}=m_{\mathrm{sym}}$} & $\mathrm{H} 200$ & 0.137 & 0.137 & 4.4 & $96 \times 32^{3}$ & 418 & 418 & 8000 \\
\hline & N202 & 0.137 & 0.137 & 6.4 & $128 \times 48^{3}$ & 410 & 410 & 3536 \\
\hline & $\mathrm{N} 203$ & 0.13708 & 0.136840284 & 5.4 & $128 \times 48^{3}$ & 345 & 441 & 6172 \\
\hline & $\mathrm{N} 200$ & 0.13714 & 0.13672086 & 4.4 & $128 \times 48^{3}$ & 283 & 461 & 6800 \\
\hline & D200 & 0.1372 & 0.136601748 & 4.2 & $128 \times 64^{3}$ & 199 & 479 & 4000 \\
\hline \multirow{3}{*}{$\hat{m}_{\mathrm{s}}=\hat{m}_{\mathrm{s}}^{\text {(phys) }}$} & N204 & 0.137112 & 0.136575049 & 5.6 & $128 \times 48^{3}$ & 351 & 544 & 2000 \\
\hline & N201 & 0.13715968 & 0.136561319 & 4.5 & $128 \times 48^{3}$ & 284 & 522 & 6000 \\
\hline & D201 & 0.137207 & 0.136546436 & 4.1 & $128 \times 64^{3}$ & 198 & 499 & 4312 \\
\hline \multicolumn{9}{|c|}{$\beta=3.7[a \approx 0.05 \mathrm{fm}], \quad \sqrt{8 t_{0}} / a \approx 8.30$} \\
\hline \multirow{2}{*}{$\bar{m}=m_{\mathrm{sym}}$} & N300 & 0.137 & 0.137 & 5.1 & $128 \times 48^{3}$ & 418 & 418 & 8188 \\
\hline & $\mathrm{J} 303$ & 0.137123 & 0.1367546608 & 4.2 & $192 \times 64^{3}$ & 257 & 473 & 2536 \\
\hline \multicolumn{9}{|c|}{$\beta=3.85[a \approx 0.0395 \mathrm{fm}], \quad \sqrt{8 t_{0}} / a \approx 10.6$} \\
\hline $\bar{m}=m_{\mathrm{sym}}$ & $\mathrm{J} 500$ & 0.136852 & 0.136852 & 5.2 & $192 \times 64^{3}$ & 404 & 404 & 3368 \\
\hline
\end{tabular}

Table 1. Details of the ensembles analyzed so far for the two trajectories to the physical point, keeping $\bar{m}$ fixed to the value at the symmetric point $\left(\bar{m}=m_{\text {sym }}\right)$ and keeping the renormalized strange quark mass $\left(\hat{m}_{\mathrm{s}}\right)$ approximately equal to the physical value $\left(\hat{m}_{\mathrm{s}}^{\text {(phys) }}\right)$. The light and strange quark hopping parameters are denoted $\kappa_{\mathrm{l}}$ and $\kappa_{\mathrm{S}}$, respectively. The lattice volumes $N_{t} \times N_{s}^{3}$, the pion $\left(m_{\pi}\right)$ and kaon $\left(m_{K}\right)$ masses and the statistics given by the number of molecular dynamics units $\left(N_{\mathrm{MD}}\right)$ are also indicated.

\section{Observables}

The pseudoscalar decay constants $f_{\mathrm{D}}$ and $f_{\mathrm{D}_{\mathrm{s}}}$ are the low-energy QCD contributions to the leptonic decays of $\mathrm{D}$ - and $\mathrm{D}_{\mathrm{s}}$-mesons. They are defined as the matrix elements

$$
\left\langle 0\left|A_{\mu}^{\mathrm{qc}}\right| \mathrm{D}_{\mathrm{q}}(p)\right\rangle=\mathrm{i} f_{\mathrm{D}_{\mathrm{q}}} p_{\mu},
$$

where $A_{\mu}^{\mathrm{qc}}=\bar{q} \gamma_{\mu} \gamma_{5} c$ is the axial vector current for quark flavours $\mathrm{q}=1($ ight $), \mathrm{s}$ and $\left|\mathrm{D}_{\mathrm{q}}(p)\right\rangle$ is a pseudoscalar meson state at momentum $p$ with quantum numbers corresponding to the $\mathrm{D}$ - (for $\mathrm{q}=1$ ) or $\mathrm{D}_{\mathrm{s}}$-meson (for $\mathrm{q}=\mathrm{s}$ ). We make use of the pseudoscalar operator $P^{\mathrm{qc}}=\bar{q} \gamma_{5} c$ to remove $O(a)$ discretization effects from the axial operator, obtaining the improved axial current

$$
A_{\mu}^{\mathrm{qc}, \mathrm{I}}=A_{\mu}^{\mathrm{qc}}+a c_{\mathrm{A}} \frac{1}{2}\left(\partial_{\mu}+\partial_{\mu}^{*}\right) P^{\mathrm{qc}},
$$

with the standard notation for lattice forward and backward derivatives. Renormalization then proceeds via

$$
\left(A_{\mu}^{\mathrm{qc}, \mathrm{I}}\right)_{\mathrm{R}}=Z_{\mathrm{A}}\left[1+a\left(b_{\mathrm{A}} m_{\mathrm{qc}}+3 \tilde{b}_{\mathrm{A}} \bar{m}\right)\right] A_{\mu}^{\mathrm{qc}, \mathrm{I}}+O\left(a^{2}\right) .
$$


Here, $m_{\mathrm{qc}}$ and $\bar{m}$ denote the bare vector Ward identity quark mass combinations

$$
m_{\mathrm{qc}}=\frac{1}{2}\left(m_{\mathrm{q}}+m_{\mathrm{c}}\right), \quad \bar{m}=\frac{1}{3}\left(m_{\mathrm{s}}+2 m_{1}\right)
$$

The improvement coefficients $c_{\mathrm{A}}$ and $Z_{\mathrm{A}}$ have been calculated non-perturbatively in Refs $[12,13] . b_{\mathrm{A}}$ has been determined non-perturbatively in Ref [14] and in a preliminary analysis the same authors find $\tilde{b}_{\mathrm{A}}$ to be consistent with zero. Since $\bar{m}$ includes only the light and strange quark, the mass dependent corrections in Eq. (5) are dominated by the charmed mass term $m_{\mathrm{qc}}$. Therefore we neglect the term proportional to $\tilde{b}_{\mathrm{A}} \bar{m}$ in our analysis.

In order to extract the matrix elements of Eq. (3), we evaluate the two-point functions

$$
C_{\mathrm{A}}\left(x_{0}, y_{0}\right)=-\frac{a^{6}}{L^{3}} \sum_{\vec{x}, \vec{y}}\left\langle A_{4}^{\mathrm{qc}, \mathrm{I}}(x)\left(P^{\mathrm{qc}}(y)\right)^{\dagger}\right\rangle, \quad C_{\mathrm{P}}\left(x_{0}, y_{0}\right)=-\frac{a^{6}}{L^{3}} \sum_{\vec{x}, \vec{y}}\left\langle P^{\mathrm{qc}}(x)\left(P^{\mathrm{qc}}(y)\right)^{\dagger}\right\rangle,
$$

at zero momentum, with $y_{0}$ being the timeslice of the source insertion and $x_{0}$ that of the sink. Starting from the spectral decomposition of the two-point functions

$$
C_{\mathrm{X}}\left(x_{0}, y_{0}\right)=\sum_{i=1}^{\infty} c_{\mathrm{X}, i} \mathrm{e}^{-E_{i}\left(x_{0}-y_{0}\right)} \text { with } E_{1}=m_{\mathrm{D}_{\mathrm{q}}}, E_{i \geq 2}: \text { excited states, } \mathrm{X}=\mathrm{P}, \mathrm{A},
$$

it can be shown [10] that for large time separations $x_{0}-y_{0}$ the correlators behave as

$$
\begin{aligned}
C_{\mathrm{A}}\left(x_{0}, y_{0}\right) & \approx \frac{f_{\mathrm{qc}}^{\text {bare }}}{2} A\left(y_{0}\right) \mathrm{e}^{-m_{\mathrm{D}_{\mathrm{q}}}\left(x_{0}-y_{0}\right)} \\
& \equiv c_{\mathrm{A}, 1}\left(y_{0}\right) \mathrm{e}^{-m_{\mathrm{D}_{\mathrm{q}}}\left(x_{0}-y_{0}\right)} \\
C_{\mathrm{P}}\left(x_{0}, y_{0}\right) & \approx \frac{\left|A\left(y_{0}\right)\right|^{2}}{2 m_{\mathrm{D}_{\mathrm{q}}}} \mathrm{e}^{-m_{\mathrm{D}_{\mathrm{q}}}\left(x_{0}-y_{0}\right)} \\
& \equiv c_{\mathrm{P}, 1}\left(y_{0}\right) \mathrm{e}^{-m_{\mathrm{D}_{\mathrm{q}}}\left(x_{0}-y_{0}\right)}
\end{aligned}
$$

where the bare decay constant $f_{\mathrm{qc}}^{\text {bare }}$ corresponds to $\left\langle 0\left|A_{4}^{\mathrm{qc}, \mathrm{I}}\right| \mathrm{D}_{\mathrm{q}}\right\rangle / m_{\mathrm{D}_{\mathrm{q}}}$, while $A\left(y_{0}\right)$ encodes the matrix element $\left\langle 0\left|P^{\mathrm{qc}}\right| \mathrm{D}_{\mathrm{q}}\right\rangle$ plus possible boundary contaminations at the source position.

\section{Analysis details}

Elaborating on our earlier status report in [2], we describe our current analysis setup in this section. It features point-to-all propagators with Wuppertal smearing [15, 16] and APE-smoothed links [17] for the pseudoscalar source and sink operator $P^{\mathrm{qc}}$. In order to gain statistics, source operators are inserted at three different positions. For $\beta=3.4$, for example, the source positions are $y_{0} / a=30,47$ and 65 .

We exploit time-reversal symmetry of the correlators and average the forward propagating part of the $C_{\mathrm{X}}\left(x_{0}, 30\right)$ correlator with source position at $y_{0} / a=30$ with the backwards propagating part of the $C_{\mathrm{X}}\left(x_{0}, 65\right)$ correlator, while for the $C_{\mathrm{X}}\left(x_{0}, 47\right)$ correlator we average the forward and backward propagating parts. This yields four correlators, two for both the axial and the pseudoscalar case. In order to determine a region where a one-state fit to the ground state can be done safely, we first perform a double-exponential fit of the form

$$
C_{\mathrm{X}}\left(x_{0}, y_{0}\right)=c_{\mathrm{X}, 1} \mathrm{e}^{-m_{\mathrm{D}_{\mathrm{q}}}\left(x_{0}-y_{0}\right)}+c_{\mathrm{X}, 2} \mathrm{e}^{-M_{\mathrm{D}_{\mathrm{q}}}^{\prime}\left(x_{0}-y_{0}\right)},
$$



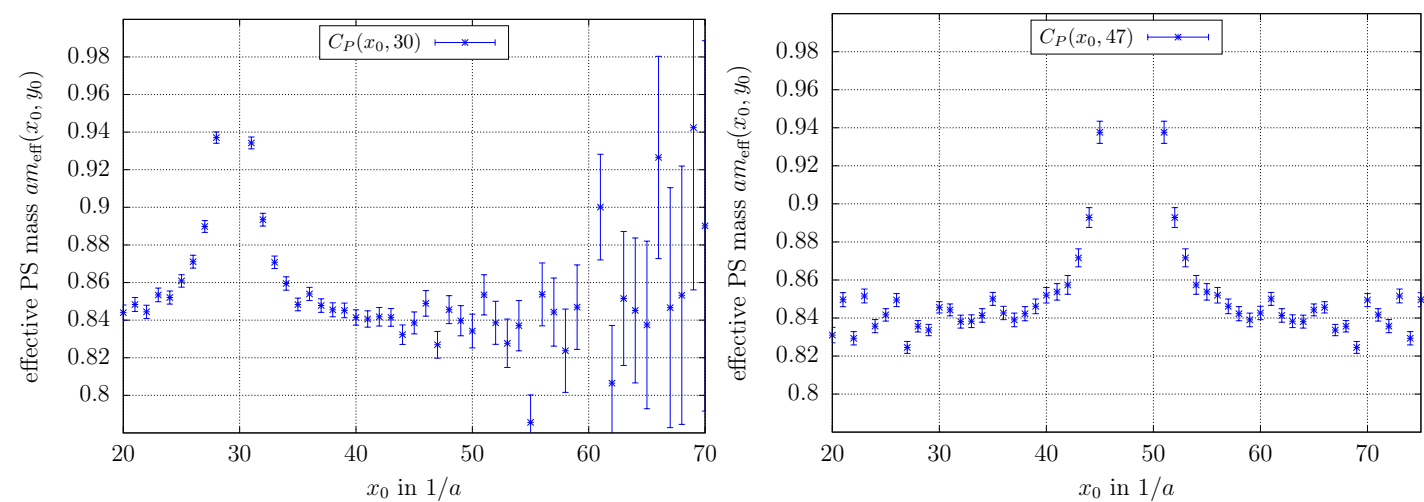

Figure 2. The effective mass of the pseudoscalar D-meson in units of $x_{0} / a$ for the H105 ensemble with $N_{t}=96$. The left panel shows the effective mass with the source positioned at $x_{0} / a=30$, while in the right panel the source is inserted at $x_{0} / a=47$.

where the second term represents the first excited state with mass $M_{\mathrm{D}_{\mathrm{q}}}^{\prime}$. At first, this is done for the pseudoscalar and axial correlators separately. Since all sources are placed far away from the boundary such that boundary contaminations are expected to be negligible, $A\left(y_{0}\right)=$ const. (see Eq. (9)) holds and the amplitudes can be enforced to be the same for different source positions (e.g., $c_{\mathrm{P}, 1}$ has the same value for both types of source-position-averaged pseudoscalar correlators). We vary both the starting point and the end point of the fit interval in order to find the optimal fit range, indicated by a minimum of the obtained $\chi^{2}$-values. In addition, a careful visual inspection of the fit quality is performed. Then the point $x_{0}^{\mathrm{min}}$, where the contributions from the exited states have sufficiently

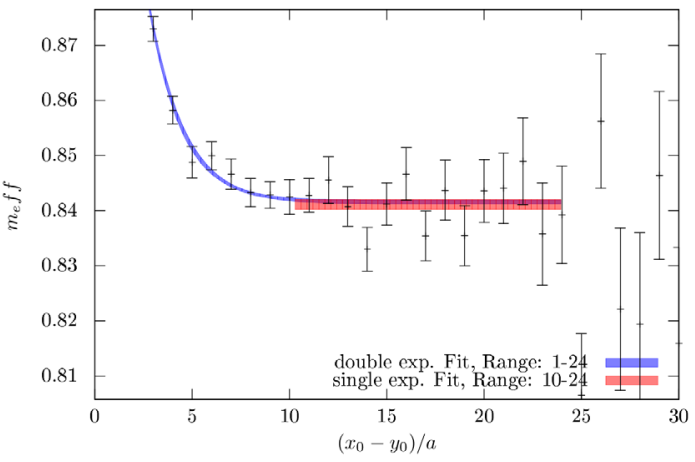

Figure 3. Effective mass of the D-meson as a function of the source-sink separation $\left(x_{0}-y_{0}\right) / a$ for the H105 ensemble. The double-exponential fit determines the starting point $x_{0}^{\min }$ of the single-exponential fit to be at $\left(x_{0}-y_{0}\right) / a=10$ in this case. decayed, is given by the criterion

$$
\frac{\left|c_{\mathrm{X}, 2}\right|^{2} \mathrm{e}^{-M_{\mathrm{D}_{\mathrm{q}}}^{\prime}\left(x_{0}^{\min }-y_{0}\right)}}{2 M_{\mathrm{D}_{\mathrm{q}}}^{\prime}}<\frac{1}{4} \Delta C_{\mathrm{X}}\left(x_{0}^{\min }, y_{0}\right),
$$

where $\Delta C_{\mathrm{X}}$ denotes the statistical error of the correlator. Finally, $x_{0}^{\min }$ is taken as the start point for a simultaneous fit of all four correlators to a single-exponential form:

$$
C_{\mathrm{X}}\left(x_{0}, y_{0}\right)=c_{\mathrm{X}, 1} \mathrm{e}^{-m_{\mathrm{D}_{\mathrm{q}}}\left(x_{0}-y_{0}\right)} .
$$

The bare pseudoscalar decay constant $f_{\mathrm{D}_{\mathrm{qc}}}^{\text {bare }}\left(\mathrm{q}=1\right.$ for the D-meson and $\mathrm{q}=\mathrm{s}$ for the $\mathrm{D}_{\mathrm{s}}-$ meson) is then given by the ratio

$$
f_{\mathrm{D}_{\mathrm{qc}}}^{\text {bare }}=\frac{\sqrt{2} c_{\mathrm{A}, 1}}{\sqrt{c_{\mathrm{P}, 1} m_{\mathrm{D}_{\mathrm{q}}}}} \text {. }
$$


For the end point $x_{0}^{\max }$ of the fit range we choose roughly the point, at which the ratio of the statistical error and mean of the correlator exceeds $3 \%$. Both the double-exponential and the singleexponential fits are illustrated in Fig. 3 for the example of the H105 ensemble.

Since our setup does not include a dynamical charm quark, $\kappa_{\text {charm }}$ has to be fixed for each ensemble. Based on a subset of statistics, we first estimate $\kappa_{\text {charm }}$ from the spin-flavour-averaged $1 \mathrm{~S}$ mass combination $M_{\mathrm{X}}=\left(6 m_{\mathrm{D}^{*}}+2 m_{\mathrm{D}}+3 m_{\mathrm{D}_{\mathrm{s}}^{*}}+m_{\mathrm{D}_{\mathrm{s}}}\right) / 12$ along the $\bar{m}=$ const. line and from the spin-averaged mass combination $M_{\mathrm{X}}=\left(3 m_{\mathrm{D}_{\mathrm{s}}^{*}}+m_{\mathrm{D}_{\mathrm{s}}}\right) / 4$ along the $\hat{m}_{\mathrm{s}}=$ const. line. Afterwards, simulations with full statistics were performed at two values of $\kappa_{\text {charm }}$ slightly above and below the estimated value, allowing for an uncertainty in the lattice spacing $a$ of $\pm 2 \%$. A linear interpolation of $M_{\mathrm{X}}$ in $1 / \kappa_{\text {charm }}$ to the physical point, given by the central value of $a$, then fixes the physical $\kappa_{\text {charm }}^{\text {(phy) }}$. The bare pseudoscalar decay constants $f_{\mathrm{D}_{(\mathrm{s})}}$ are then interpolated linearly to this value. Linear interpolations are valid here, since for each ensemble the two chosen values of $\kappa_{\text {charm }}$ are sufficiently close to the target $\kappa_{\text {charm }}^{\text {(phys) }}$.

\section{Preliminary results}

The results described in this section represent the status of the project at the time of the conference and are summarized in Figs. 4 and 5. Statistical error analyses are performed using bootstrap techniques, where the bin size is varied to perform an extrapolation of the error to infinite bin size, as well as adopting the so-called $\Gamma$-method, which extracts the statistical errors from the evaluation of autocorrelation functions [18]. Possible sources of systematic errors have still to be accounted for in the final analysis.
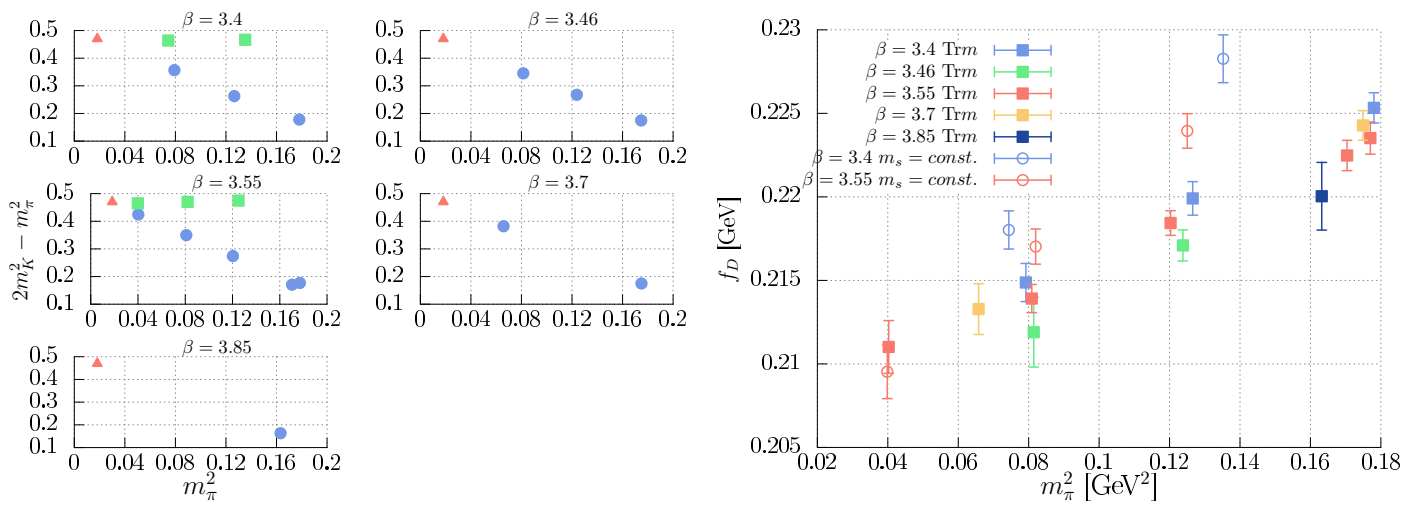

Figure 4. Ensembles analyzed so far (left panel) and preliminary results for $f_{\mathrm{D}}$ as a function of $m_{\pi}^{2}$ (right panel).

As the left panel of Fig. 4 shows, the majority of ensembles at our disposal has been included in the analysis so far. Ensembles for the $\bar{m}=$ const. line are now available for every lattice spacing (drawn in blue), while for the $\hat{m}_{\mathrm{s}}=$ const. line ensembles have been included for $\beta=3.4$ and $\beta=3.55$ only (drawn in green). The physical point in the quark mass plane is indicated by a red triangle. Results for the pseudoscalar decay constant $f_{\mathrm{D}}$ are presented in the right panel of Fig. 4, as a function of $m_{\pi}^{2}$. The left panel of Fig. 5 shows the corresponding results in the case of $f_{\mathrm{D}_{\mathrm{s}}}$, while in the right panel the ratio $f_{\mathrm{D}_{\mathrm{s}}} / f_{\mathrm{D}}$ is presented along with the recent FLAG [1] averages for $N_{\mathrm{f}}=2+1$ and $N_{\mathrm{f}}=2+1+1$ at the physical point. Combined linear fits in the right panel do not yet represent a full chiral and continuum extrapolation, but have been included in order to demonstrate consistency with the FLAG 

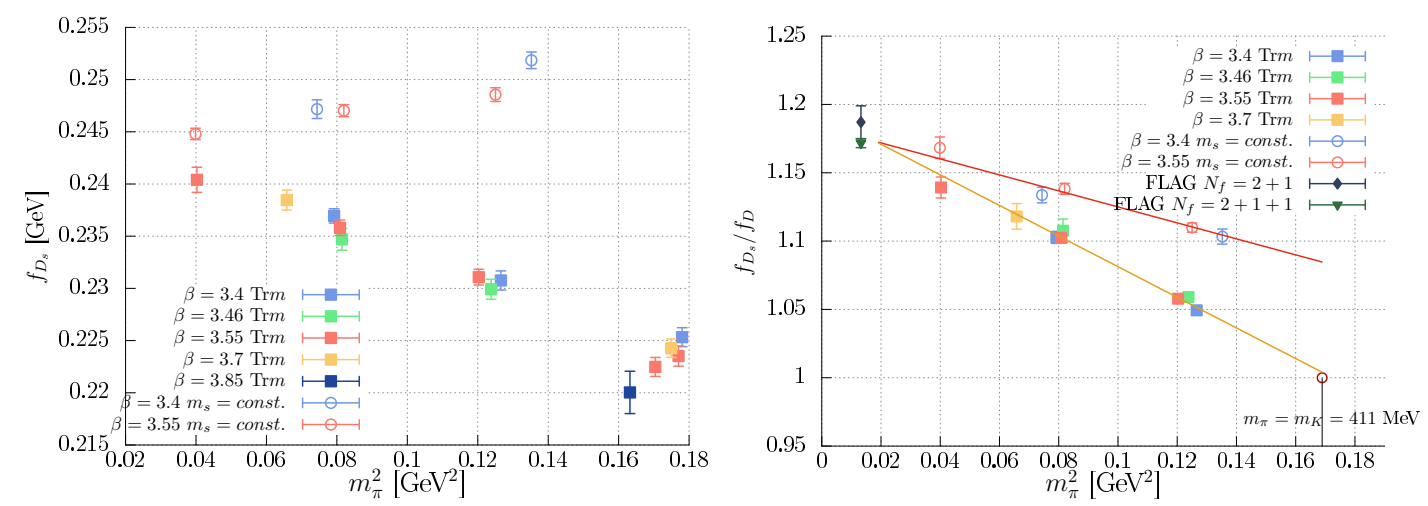

Figure 5. Preliminary results for $f_{\mathrm{D}_{\mathrm{s}}}$ (left panel) and the ratio $f_{\mathrm{D}_{\mathrm{s}}} / f_{\mathrm{D}}$ as a function of $m_{\pi}^{2}$ (right panel). The linear fit in the right panel was done to guide the eye and does not represent a full chiral and continuum extrapolation.

results. Along the $\bar{m}=$ const. line, the ratio of $f_{\mathrm{D}_{\mathrm{s}}} / f_{\mathrm{D}}$ is expected to be one at the symmetric point $m_{1}=m_{\mathrm{s}}$, which is also in agreement with the linear fits employed. This finding, in conjunction with the observed absence of large discretization and finite-size effects, leads us to conclude that these preliminary estimates of the $\mathrm{D}_{(\mathrm{s})}$-meson decay constants in three-flavour lattice QCD are intermediate promising results.

\section{Outlook}

In order to improve on the precision of our measurements, we will continue to increase statistics, as well as to perform a careful analysis of the statistical errors. Further steps still to be done include gaining a good control over systematic effects and performing combined chiral and continuum extrapolations. Furthermore, a cross check of the results via a second method of extracting the decay constants, utilizing the axial Ward identity (PCAC) quark mass [19], is being prepared. An investigation of the size of the effect from a slight mistuning of the quark mass trajectories [7, 10] is also planned.

\section{Acknowledgments}

We thank Gunnar Bali, Tomasz Korzec, Stefan Schaefer and Rainer Sommer for useful discussions. This work is supported by the Deutsche Forschungsgemeinschaft (DFG) through the grants GRK 2149 (Research Training Group "Strong and Weak Interactions - from Hadrons to Dark Matter”, K. E. and J. H.) and the SFB/TRR 55 (S. C., S. H. and W. S.). We are indebted to our colleagues in CLS for the joint production of the $N_{\mathrm{f}}=2+1$ gauge configurations. The authors gratefully acknowledge the Gauss Centre for Supercomputing e.V. for granting computer time on SuperMUC at the Leibniz Supercomputing Centre. Additional simulations were performed on the Regensburg iDataCool cluster and on the SFB/TRR 55 QPACE 2 and QPACE B computers [20, 21]. The two-point functions were computed using the Chroma [22] software package, along with the locally deflated domain decomposition solver implementation of openQCD [3], the LibHadronAnalysis library and the multigrid solver implementation of Ref. [23]; additional calculations were carried out using the code based on [24]. 


\section{References}

[1] S. Aoki et al., Eur. Phys. J. C77, 112 (2017), 1607.00299

[2] S. Collins, K. Eckert, J. Heitger, S. Hofmann, W. Söldner (ALPHA, RQCD), PoS LATTICE2016, 368 (2017), 1701.05502

[3] M. Lüscher, S. Schaefer, http://luscher.web.cern.ch/luscher/openQCD/ (2016 (accessed Nov 14, 2016))

[4] M. Lüscher, S. Schaefer, JHEP 07, 036 (2011), 1105 . 4749

[5] M. Lüscher, S. Schaefer, Comput. Phys. Commun. 184, 519 (2013), 1206.2809

[6] M. Lüscher, F. Palombi, PoS LATTICE2008, 049 (2008), 0810. 0946

[7] M. Bruno et al., JHEP 02, 043 (2015), 1411. 3982

[8] G.S. Bali, E.E. Scholz, J. Simeth, W. Söldner (RQCD), Phys. Rev. D94, 074501 (2016), 1606.09039

[9] W. Bietenholz et al., Phys. Lett. B690, 436 (2010), 1003 . 1114

[10] M. Bruno, T. Korzec, S. Schaefer, Phys. Rev. D95, 074504 (2017), 1608.08900

[11] S. Borsanyi et al., JHEP 09, 010 (2012), 1203. 4469

[12] J. Bulava, M. Della Morte, J. Heitger, C. Wittemeier (ALPHA), Nucl. Phys. B896, 555 (2015), 1502.04999

[13] J. Bulava, M. Della Morte, J. Heitger, C. Wittemeier (ALPHA), Phys. Rev. D93, 114513 (2016), 1604.05827

[14] P. Korcyl, G.S. Bali, Phys. Rev. D95, 014505 (2017), 1607.07090

[15] S. Güsken, U. Löw, K. Mütter, R. Sommer, A. Patel, K. Schilling, Phys. Lett. B227, 266 (1989)

[16] S. Güsken, Nucl. Phys. B Proc. Suppl. 17, 361 (1990)

[17] M. Falcioni, M. Paciello, G. Parisi, B. Taglienti, Nucl. Phys. B251, 624 (1985)

[18] U. Wolff (ALPHA), Comput. Phys. Commun. 156, 143 (2004), hep-lat/0306017

[19] J. Heitger, G.M. von Hippel, S. Schaefer, F. Virotta (ALPHA), PoS LATTICE2013, 475 (2014), 1312.7693

[20] H. Baier et al., PoS LAT2009, 001 (2009), 0911.2174

[21] Y. Nakamura, A. Nobile, D. Pleiter, H. Simma, T. Streuer, T. Wettig, F. Winter (2011), 1103.1363

[22] R.G. Edwards, B. Joó (SciDAC, LHPC, UKQCD), Nucl. Phys. Proc. Suppl. 140, 832 (2005), hep-lat/0409003

[23] S. Heybrock, M. Rottmann, P. Georg, T. Wettig, PoS LATTICE2015, 036 (2016), 1512.04506

[24] T. Korzec, https://github.com/to-ko/mesons (2014) 